\title{
Population genomic analysis of Cryptococcus Brazilian isolates reveals an African type subclade distribution
}

\author{
Corinne Maufrais, ${ }^{1,2, \dagger}$ Luciana de Oliveira, ${ }^{1, \dagger}$ Rafael W. Bastos, ${ }^{3}$ Frédérique Moyrand, ${ }^{1}$ Flavia C. G. Reis, ${ }^{4,5}$ Clara Valero (DD , ${ }^{3}$ \\ Bianca Gimenez, ${ }^{4}$ Luisa J. Josefowicz, ${ }^{4}$ Gustavo H. Goldman, ${ }^{3}$ Marcio L. Rodrigues, ${ }^{4,6}$ and Guilhem Janbon (D) ${ }^{1, *}$ \\ ${ }^{1}$ Unité Biologie des ARN des Pathogènes Fongiques, Département de Mycologie, Institut Pasteur, F-75015 Paris, France \\ ${ }^{2}$ Institut Pasteur, HUB Bioinformatique et Biostatistique, C3BI, USR 3756 IP CNRS, F-75015 Paris, France \\ ${ }^{3}$ Departamento de Ciências Farmacêuticas, Faculdade de Ciências Farmacêuticas de Ribeirão Preto, Universidade de São Paulo, $14040-903$ Ribeirão Preto, Brazil \\ ${ }^{4}$ Instituto Carlos Chagas, Fundação Oswaldo Cruz (FIOCRUZ), 81310-020 Curitiba, Brazil \\ ${ }^{5}$ Centro de Desenvolvimento Tecnologico em Saude (CDTS-Fiocruz), 21040-361 Rio de Janeiro, Brazil \\ ${ }^{6}$ Instituto de Microbiologia Paulo de Góes (IMPG), Universidade Federal do Rio de Janeiro, 21941-902 Rio de Janeiro, Brazil
}

*Corresponding author: janbon@pasteur.fr

${ }^{\dagger}$ Both authors should be considered as first authors.

\begin{abstract}
The genomes of a large number of Cryptococcus neoformans isolates have been sequenced and analyzed in recent years. These genomes have been used to understand the global population structure of this opportunistic pathogen. However, only a small number of South American isolates have been considered in these studies, and the population structure of $C$. neoformans in this part of the world remains elusive. Here, we analyzed the genomic sequences of 53 Brazilian Cryptococcus isolates and deciphered the C. neoformans population structure in this country. Our data reveal an African-like structure that suggested repeated intercontinental transports from Africa to South America. We also identified a mutator phenotype in one VNBII Brazilian isolate, exemplifying how fast-evolving isolates can shape the Cryptococcus population structure. Finally, phenotypic analyses revealed wide diversity but not lineage specificity in the expression of classical virulence traits within the set of isolates.
\end{abstract}

Keywords: Cryptococcus neoformans; Cryptococcus gattii; Cryptococcus deuterogattii; genome; Brazil; phylogenetic tree

\section{Introduction}

The current classification identifies seven human-pathogenic Cryptococcus species, which are responsible for 180,000 deaths every year in the world (Hagen et al. 2015; Rajasingham et al. 2017). Cryptococcus neoformans and Cryptococcus deneoformans represent the neoformans group of species, whereas the gattii group of species comprises Cryptococcus gattii, Cryptococcus deuterogattii, Cryptococcus tetragattii, Cryptococcus decagattii, and Cryptococcus bacillisporus (Hagen et al. 2015). Cryptococcus neoformans is the most common species in clinical settings, representing more than $95 \%$ of the clinical isolates (Kwon-Chung et al. 2014; Janbon et al. 2019). This species affects mostly immunocompromised patients in whom it provokes meningoencephalitis. Other species, such as C. gattii, can be primary pathogens and cause pulmonary infections (Kwon-Chung et al. 2014). Cryptococcus neoformans is thought to be acquired very early in life and can stay in a dormant state for years, probably in alveolar macrophages (Garcia-Hermoso et al. 1999; Alanio 2020). As soon as a defect in the cellular immunity appears, yeast cells can multiply, reach the central nervous system, and induce meningoencephalitis, which is invariably deadly if untreated.

Cryptococcus neoformans strains are ubiquitous in the environment and have been isolated from all continents except
Antarctica. Similarly, although isolates of the gattii group of species have been long considered to be geographically restricted to tropical and subtropical regions and typically associated with Eucalyptus trees, they have emerged as a primary pathogen in western Canada and the United States of America (Kidd et al. 2004). Both species can live in a broad range of ecological habitats and have been commonly isolated from soil, guano, decaying wood, fruits, and insects (Lin and Heitman 2006). Pigeons (Columba livia) are vectors for the worldwide dissemination of Cryptococcus. Indeed, dry pigeon guano seems to be an ideal environment for C. neoformans development and is commonly infected with this fungus. Nevertheless, pigeons are usually not harmed by $\mathrm{C}$. neoformans because their body temperature is too high $\left(42.5^{\circ} \mathrm{C}\right)$ for its growth and disease development (Johnston et al. 2016).

All neoformans and gattii species are haploid species with two possible mating-type alleles (MATa and MAT $\alpha$ ) and a bipolar mating system. Efficient sexual reproduction has also been described in the laboratory. Nevertheless, C. neoformans MATa isolates are rare in clinical and environmental isolates with the exception of some places in Africa (Kwon-Chung and Bennett 1978; Litvintseva et al. 2003). In addition, it has been reported that $8 \%$ of the population of $\mathrm{C}$. neoformans isolates are diploid (Lin et al. 
2009). Diverse hybrids have also been isolated from environmental and clinical sources. The most common are AD hybrids, which are produced by hybridization of $C$. neoformans with $C$. deneoformans isolates. In addition, rare cases of gattii/neoformans hybrids have been reported (Bovers et al. 2008; Aminnejad et al. 2012). Nevertheless, population structure analysis suggested that the different neoformans and gattii populations are largely clonal with a limited degree of recombination (Litvintseva et al. 2005; Bui et al. 2008; Litvintseva and Mitchell 2012).

Historically, these analyses were based on serotyping, which was first replaced by PCR-based characterization (RAPD, AFLP, and mating type analysis) of the isolates and then by sequencing of a defined set of genes by multilocus sequence typing (MLST) (Ruma et al. 1996; Boekhout et al. 2001; Matsumoto et al. 2007; Meyer et al. 2009). Originally, five neoformans molecular subtypes were thus defined (VNI, VNII, VNIV, VNIII, and VNB) (Meyer et al. 2009). The new species definition includes VNI, VNII, and VNB within C. neoformans, VNIV within C. deneoformans, and VNIII as C. neoformans/C. deneoformans hybrids. VNI is the most common C. neoformans molecular subtype and has a global distribution. The VNB subtype was discovered in Africa and has a genetically variable population with $10 \%$ MATa isolates, which are very rare in all other studied populations (Litvintseva et al. 2003). The VNB clade includes strains isolated from the endemic mopane tree (Colophospermum mopane), whereas strains isolated from pigeon excreta in the same area had the VNI molecular type, which is shared by most isolates globally. This observation supported the "out of Africa" hypothesis in which few of these isolates would have adapted their biology to multiply in pigeons to achieve global dispersion (Litvintseva et al. 2011; Litvintseva and Mitchell 2012).

More recently, the improvement of sequencing technologies and bioinformatics tools allowed analysis of whole-genome sequences, revealing new details in the structure and global geographical distribution of the Cryptococcus populations together with the identification of additional clades and subclades (Farrer et al. 2019). Most of these studies focused on C. neoformans, with $>1000$ C. neoformans genome sequences analyzed in four studies (Desjardins et al. 2017; Rhodes et al. 2017b; Vanhove et al. 2017; Ashton et al. 2019; Ergin et al. 2019). Through these efforts, the VNB clade has been separated into the VNBI and VNBII subclades, which have different resistance to oxidative stress and a different ability to produce melanin (Desjardins et al. 2017; Vanhove et al. 2017). The VNI clade has been separated into a number of subclades with three phylogenetic groups representing the majority of the studied isolates (Rhodes et al. 2017b; Ashton et al. 2019). Surprisingly, only 11 haploid strains isolated in South America were included in these studies. This small number conflicts with the relevance of South America as a source of genetic diversity in the gattii isolates (Engelthaler et al. 2014). Up until now, the only analysis of C. neoformans population structure in South America has been an MLST analysis that concluded that subtype ST93 was the most prevalent among strains isolated from HIV patients in Southeastern Brazil with a low level of recombination (Ferreira-Paim et al. 2017). Similarly, few phenotypic analyses that include South American isolates have been published, and a recent analysis of virulence factor expression within Brazilian Cryptococcus clinical isolates does not include any sequencing or molecular typing analysis (De Sousa et al. 2020). In this study, we analyzed the phenotypes and genome sequences of 53 Brazilian isolates, revealing very high phenotypic diversity within Cryptococcus isolates and a subclade distribution that suggests several waves of Cryptococcus introduction from Africa to South America.

\section{Materials and methods Isolates used in this study}

Strains 2B, 2C, 2D, 4C, 7, 33A1, 54A1, 56A1, 68B1, 69A1, 81A1, 96A1, 106A1, 144A1, 151A1, 176A1, 3Pb3, 5Pb2, 19Pb4, 23Pb2, 32Bp1, and RR 2605 were isolated from Northern Brazil (Dal Pupo et al. 2019) and kindly donated by Dr. Halan Dal Pupo. Strains Cn201, Cn359, Cn894, Cn14HC, and Cn201 were obtained from the culture collection of the Central Hospital of the University of São Paulo. All other isolates were obtained from the Collection of Pathogenic Fungi available at Fiocruz. No genomic information was available for any of these isolates before the present study.

\section{Read alignment, variant detection, and ploidy analysis}

Illumina reads were aligned to the Cryptococcus reference genomes using Minimap2 aligner v. 2.9 (Li 2018) with the "-ax sr" parameter. BAM files were sorted and indexed using SAMtools (Li et al. 2009) version 1.9. Picard version 2.8.1 (http://broadinstitute. github.io/picard) tools were used to identify duplicate reads and assign correct read groups to BAM files. SAMtools version 1.9 and Picard version 2.8.1 were then used to filter, sort, and convert SAM files and assign read groups and mark duplicate reads. Single-nucleotide polymorphisms (SNPs) and insertions/deletions (indels) were called using Genome Analysis Toolkit version 3.6 with ploidy $=1$ according to the GATK Best Practices. HaploScore parameters used to filter SNPs and indels included VariantFiltration, QD $<2.0$, LowQD, ReadPosRankSum<-8.0, LowRankSum, FS >60.0, HightFS, MQRankSum<-12.5, MQRankSum, MQ <40.0, LowMQ, and HaplotypeScore >13.0. Phyml version 20120412 (Guindon et al. 2010) with the GTR, NNIs substitution model was used to infer phylogenetic relationships between the isolates using the separate data set of confident SNPs. To examine variations in ploidy across the genome, the sequencing depth at all positions was computed using SAMtools (Li et al. 2009), and then the average depth was computed for 1-kb windows across the genome.

\section{MAT locus determination}

The mating type of Brazilian isolates was determined as previously published (Rhodes et al. 2017b). Briefly, Illumina reads were aligned to the mating-type locus sequences (AF542529.2 and AF542528.2) using BWA-MEM. Computed average depths at the SXI and STE2O loci using SAMtools mpileup were used to determine the mating type.

\section{Population inference by fastSTRUCTURE}

We used fastSTRUCTURE (Raj et al. 2014) to identify admixture in the data set from $K=2$ to 4 . After $K=4$, clusters with the highest number of isolates were split into subclusters, which likely reflects an issue due to the number of isolates within clusters rather than true biological significance.

\section{Drug susceptibility testing}

The minimum inhibitory concentration (MIC) of fluconazole (FLC) (Sigma-Aldrich) was determined as described by M27-A3 (Clinical Laboratory Standards Institute 2017). 


\section{Titan cell production}

Titan cell (TC) production was evaluated as described by Dambuza et al. (2018) with some modifications. The isolates were cultivated in yeast peptone dextrose (YPD) agar (1\% yeast extract, $2 \%$ bacto-peptone, $2 \%$ glucose, and $2 \%$ bacto-agar) for 48 hours at $30^{\circ} \mathrm{C}$. A single colony was transferred to $5 \mathrm{ml} \mathrm{YNB}$ without amino acids (Sigma Y1250) prepared according to the manufacturer's instructions plus $2 \%$ glucose. After incubation overnight at $30^{\circ} \mathrm{C}$ and $200 \mathrm{rpm}, 1 \times 10^{3}$ cells $/ \mathrm{ml}$ were inoculated into $5 \mathrm{ml} 1 \times \mathrm{PBS}$ (phosphate buffer solution) $+10 \%$ heat-inactivated (HI) fetal calf serum (FCS) and incubated for 72 hours at $37^{\circ} \mathrm{C}, 5 \% \mathrm{CO}_{2}$. We visualized the cells microscopically without any staining, and 50 cells were measured. TCs were considered body cells (without the capsule) with a diameter $>10 \mu \mathrm{m}$. The experiment was repeated twice.

\section{Capsule staining}

Fungal cells were cultivated in YPD agar for 24 hours at room temperature. Single colonies were transferred by sterile loop to the wells of a 96-well plate, each containing $200 \mu$ l of Roswell Park Memorial Institute (RPMI) medium at a final cell density of $10^{7}$ yeast $/ \mathrm{ml}$. The plates were incubated for an additional 24 hours at $37^{\circ} \mathrm{C}$ in $5 \% \mathrm{CO}_{2}$ and centrifuged to separate the cells from the supernatants. The supernatants were stored for GXM analysis, and the cells were washed twice in PBS for further fixation in $4 \%$ paraformaldehyde for 1 hour at room temperature. Yeast cells were then blocked [1\% bovine serum albumin (BSA) in PBS for 1 hour at $37^{\circ} \mathrm{C}$ ] and incubated with the $18 B 7$ anti-GXM monoclonal antibody (Casadevall et al. 1992) (mAb 18B7; $10 \mu \mathrm{g} / \mathrm{ml}$ for 1 hour at $37^{\circ} \mathrm{C}$ ), a kind gift from Dr. Arturo Casadevall (Johns Hopkins University). The cells were washed and incubated with an Alexa Fluor 488-conjugated secondary antibody $(10 \mu \mathrm{g} / \mathrm{ml}$ for 1 hour at $37^{\circ} \mathrm{C}$; Invitrogen, USA). The cells were finally stained with calcofluor white $\left(5 \mu \mathrm{g} / \mathrm{ml}\right.$ for 1 hour at $37^{\circ} \mathrm{C}$; Invitrogen, USA). Alternatively, the cells were counterstained with India ink for capsular visualization. The cells were visualized on a Leica TCS SP5 confocal microscope or analyzed with a FACS Canto II flow cytometer. Data were processed with the FACSDiva software, version 6.1.3.

\section{Data availability}

All sequence data from this study have been submitted to GenBank under the BioProject identification no. PRJNA702892. Supplementary files are available at figshare: https://doi.org/10. $25387 / g 3.14345426$.

\section{Results Description of the origin of the Brazilian isolates}

We selected 53 Cryptococcus Brazilian isolates, of which 12 were classified as C. gattii and 41 as C. neoformans. More than $41 \%(n=22)$ were environmental isolates and $36 \%(n=19)$ were clinical isolates. The origin of the isolate was unknown for the remaining $23 \%(n=12)$. Twenty-one strains were isolated from pigeon excreta in Northern Brazil (Dal Pupo et al. 2019), 28 isolates were obtained from the culture collections available at Fiocruz, and 4 were acquired from the Central Hospital of the University of São Paulo (Supplementary Table S1).

\section{Genome characterization}

Whole genome sequencing was performed for each of the 53 Brazilian isolates considered in this study. Between 2.7 and 10.9 million 100-nt paired-end reads were obtained and aligned to eight Cryptococcus reference genomes using Minimap2 (Li 2018) (Supplementary Table S1). Reference genomes were C. neoformans strain H99 (Janbon et al. 2014), C. deneoformans strain JEC21 (Loftus et al. 2005; Gonzalez-Hilarion et al. 2016), C. deuterogattii strain R265 (Yadav et al. 2018; Gröhs Ferrareze et al. 2021), C. gattii WM276, C. bacillisporus strains CA1280 and CA1873, C. decagattiii strain AFLP10, and C. tetragattii strain IND107 (Basenko et al. 2018). Of the 53 isolates, 40 were identified as C. neoformans with $>80 \%$ of the reads aligning to the $\mathrm{H} 99$ genome $(<40 \%$ of reads could be aligned to any other reference genome). Among the 13 remaining isolates, 8 were $C$. deuterogattii and 5 were C. gattii (Supplementary Table S1). Of note, we found that isolate Cg366, which was previously identified as C. gattii, is actually a C. neoformans isolate. Isolates Cn201 and Cn894, which were previously considered to be C. neoformans, were identified as C. deuterogattii isolates.

In order to determine mating type, we compared the number of reads aligned to the STE20a, STE20alpha, SXI1, and SXI2 genes of the corresponding species. These analyses revealed that all isolates were MAT $\alpha$, confirming the overwhelming dominance of this mating type in clinical and environmental isolates observed in most areas in the world (Desjardins et al. 2017).

The analysis of the read alignment profiles provided insight into possible large duplications or aneuploidies. All C. neoformans isolates displayed an even alignment profile. This observation together with the absence of heterozygous SNPs in our analysis suggested that these isolates are either haploid or euploid homozygotes. However, we observed duplication of the end of chromosome 1 in three isolates (Cn160, Cn161, and Cn186) (left side in Figure 1). The isolate $\mathrm{Cn} 161$ apparently had additional duplications within chromosome 8. Similar patterns were observed in the eight C. deuterogattii isolates, with two isolates (Cn201 and Cn894) displaying a duplication of a region located in chromosomes 8 and 13, respectively. In contrast, all five C. gattii isolates displayed an even alignment profile, suggesting that all are haploid (data are not shown).

\section{Phylogenetic analyses}

To obtain insight into the population structure of the Brazilian C. neoformans isolates, we constructed a phylogenetic tree including 352 isolates considered in previous studies (Desjardins et al. 2017; Rhodes et al. 2017b; Ashton et al. 2019) that encompass all VNI and VNB subclades (Supplementary Table S2). We also included 10 VNII isolates as the outgroup to root the tree (Desjardins et al. 2017; Rhodes et al. 2017b; Ashton et al. 2019). Overall, 945,565 variable positions were used to construct the tree presented in Figure 2A.

\section{VNB isolates}

Whereas the vast majority $(92.5 \%, n=37)$ of the C. neoformans Brazilian isolates belonged to the VNI clade, we also identified one VNB isolate of the VNBII subclade (Cn225). Two other Brazilian isolates (Cn216 and $\mathrm{Cn} 14 \mathrm{HC}$ ) appear to be associated with the VNBI and VNBII clades, respectively, but with a close-tothe-root long branch (Figure 2A). Accordingly, the number of SNPs compared to the H99 reference genome was slightly higher in these two isolates than in the other VNB isolates (Figure 2B). As previously reported, analysis of the population using 


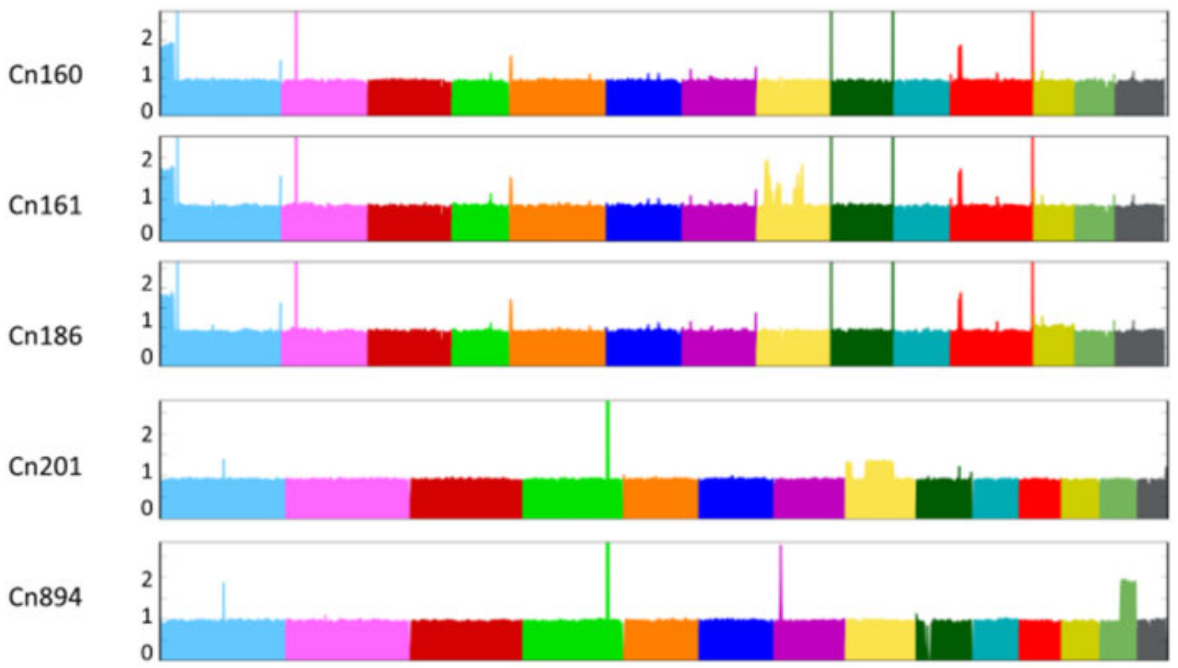

Figure 1 Large segmental duplications within the genomes of Brazilian isolates were revealed after aligning sequencing reads to the Cryptococcus reference genomes.

A

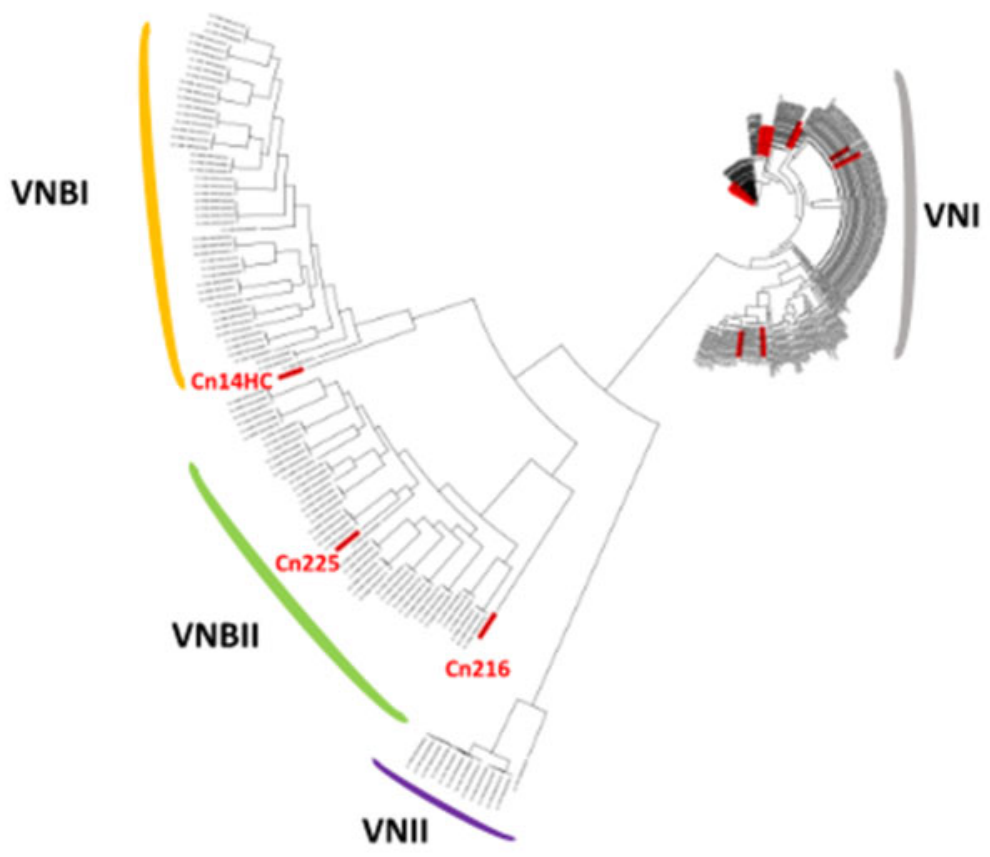

B

C

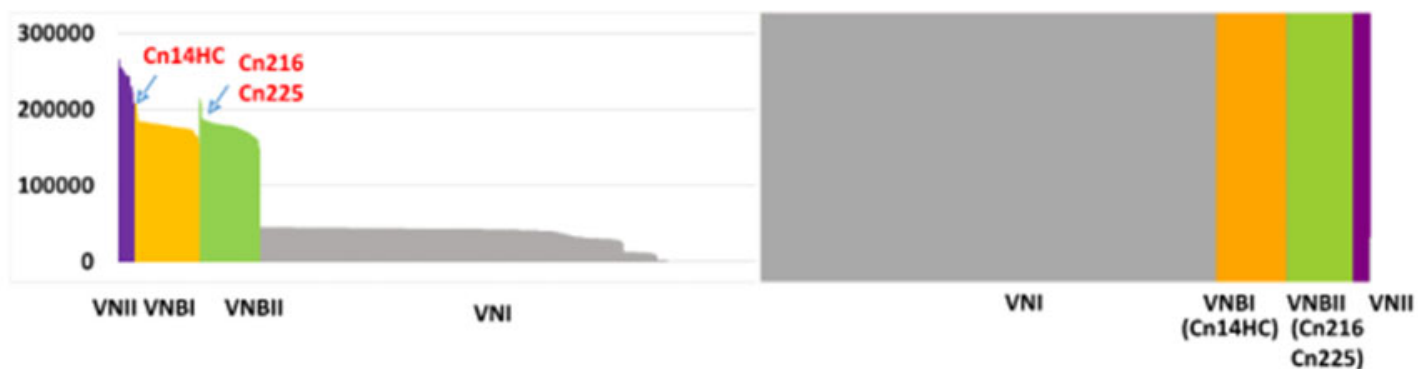

Figure 2 (A) Phylogenetic tree generated by iTOL v5 using C. neoformans genome sequences. The Brazilian isolates are indicated in red. The two Brazilian outlier isolates are shown. (B) Number of SNPs within the genomes of the different isolates analyzed in this study. (C) Results of the structure analysis $(k=4)$ suggesting VNBI and VNBII origin for the two Brazilian outlier isolates (CnH14C and Cn216, respectively). 
fastSTRUCTURE $(k=4)$ (Raj et al. 2014) readily separated VNI, VNII, VNBI, and VNBII isolates (Desjardins et al. 2017) into four independent populations (Figure 2C). As expected, this analysis showed that the two Brazilian outlier isolates (Cn216 and $\mathrm{Cn} 14 \mathrm{HC}$ ) are associated with the VNBI and VNBII clades, respectively.

Because VNI/VNB and VNII/VNB hybrids have been previously reported within African isolates (Rhodes et al. 2017b), we sought to compare their phylogenetic position with the two outlier Brazilian isolates. We first redrew a phylogenetic tree including six of these isolates [the VNII/VNB isolate MW-RSA852 and four VNI/VNB isolates (Bt125, Bt131, Bt162, and Bt163)] (Supplementary Figure S1). As previously published, the African hybrid isolates are also positioned at the root of the VNB and VNII branches, respectively. We then performed principal component analysis (PCA) including this set of hybrid isolates. As previously published, this analysis readily separated isolates positioned at an intermediary position between the defined clades (Figure 3; Desjardins et al. 2017). Like most African VNI/ VNB hybrids, the Bt163 isolate was positioned between VNI and VNBII, suggesting that it is a hybrid of isolates of these two clades. Of note, the VNI/VNB isolate Bt125 has an intermediary position between the VNBI, VNBII, and VNI lineages (Figure 3).

The two outlier Brazilian isolates are positioned between the VNBI and VNBII isolates, suggesting that they are not VNB/VNI hybrids. In agreement with the location of these isolates in the phylogenetic tree, this analysis showed that the Cn216 isolate is closer to VNBII isolates whereas the Cn14HC isolate is closer to VNBI isolates. According to these results, Cn216 and CnH14C could be either VNBI/VNBII hybrids or evolved VNBI and VNBII isolates, respectively. In order to test these two hypotheses, we first determined the position and number of SNPs specific to each clade (Figure 4, A and B). This analysis revealed that the number of clade-specific SNPs was equivalent for each clade with an even distribution along the $\mathrm{H} 99$ reference genome. Of note, the VNBI clade appears to have fewer clade-specific SNPs, which might be related to the previously reported loss of diversity in this lineage (Desjardins et al. 2017).

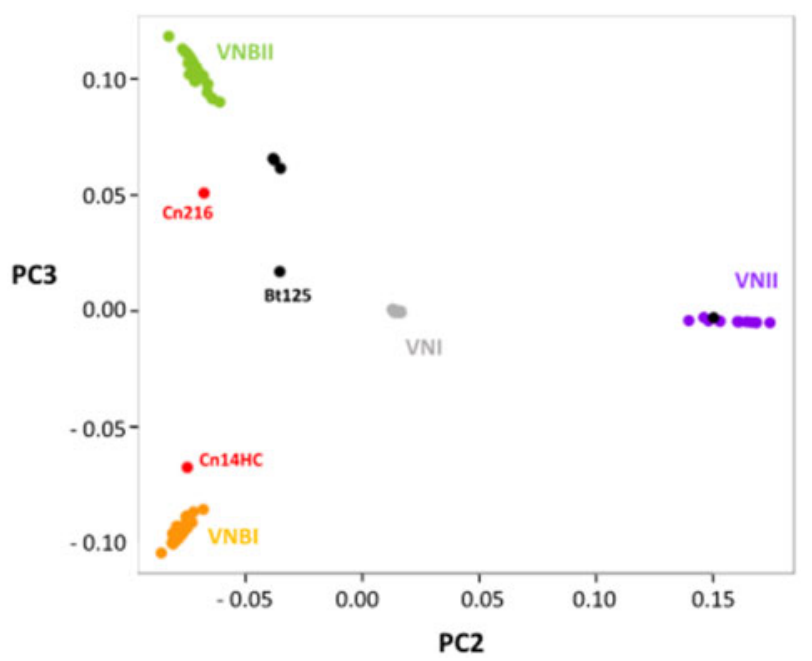

Figure 3 Principal component analysis revealing the intermediary position of the Brazilian outlier isolates. VNI/VNB and VNII/VNB hybrid isolates (Rhodes et al. 2017b) have been added to this analysis. The positions of the hybrid (black) and the two Brazilian outlier isolates (in red) are indicated.
We reasoned that we could determine the origins of the SNPs in the two outlier Brazilian isolates by comparing them with cladespecific SNPs (Figure 5). We compared the SNPs of Cn216 and Cn14HC SNPs ( $n=213,915$ and 207,438, respectively) with the VNI-, VNII-, VNBI-, and VNBI-specific SNPs and plotted their positions in the H99 reference genome. As a control, we performed the same analysis with the VNBII Brazilian isolate Cn215 ( $n=207,639$ SNPs).

This analysis revealed that the Brazilian outlier isolates Cn214 and Cn14HC mostly share SNPs with the VNBII- and VNBIspecific SNPs, respectively, confirming that they belong to these respective lineages. We observed an even distribution of these SNPs along the chromosomes with no sign of a recent recombination event between the VNBI and VNBII lineages. As expected, a similar result was obtained with the Brazilian VNBII isolate Cn225. In contrast, the African VNB/VNI hybrid Bt163 displays a SNP distribution common to the clade-specific SNPs, supporting a recent recombination event between a VNBII isolate and a VNI isolate and thus confirming the PCA results and previously published results (Rhodes et al. 2017b). For the Bt125 isolate, the SNP pattern was more complex than previously published, suggesting a triple VNBI/VNBII/VNI origin for this isolate, in agreement with the intermediary position of this isolate observed by PCA (Figure 3). Overall, these analyses suggest that the Cn214 and CnH14C isolates belong to the VNBII and VNBI clades, respectively, with no sign of recombination with another clade. Yet, the number of the SNPs unique to these two isolates $(n=37,236$ in Cn216 and $n=26,925$ in CnH14C) is much higher than in the VNBII Brazilian isolate Cn225 $(n=4788)$ and in the two VNI/VNB African hybrids $(n=7485$ in Bt125 and $n=3511$ in Bt163) (Figure 4A). These isolate-specific SNPs are evenly distributed along the H99 reference genome (Figure 4B). This SNP pattern may be due to an ancient separation of these isolates from most other isolates of their respective clades or a recent but rapid evolution of their genome sequences due to an increased mutation rate.

In order to test these hypotheses, we compared the mutation rates in both $\mathrm{Cn} 216$ and $\mathrm{Cn} 14 \mathrm{HC}$ by observing the appearance of spontaneous 5-fluorouracil (FU)-resistant colonies in fluctuation assays (Boyce et al. 2017; Billmyre et al. 2020). We included the reference strain KN99 $\alpha$ and the Brazilian VNBII isolate Cn225 as controls. The mutation rate of $\mathrm{Cn} 14 \mathrm{HC}$ was higher than those of Cn225 and KN99 $\alpha$ (Figure 6). In contrast, the mutation rate in Cn216 was not significantly different from that of Cn225 (VNBII). Similar results were obtained when 5-FOA selection was used (Supplementary Figure S2).

Isolates with mutations in the mismatch repair pathway associated with a mutator phenotype have been observed both in C. deuterogattii (Billmyre et al. 2017) and C. neoformans clinical isolates (Boyce et al. 2017; Rhodes et al. 2017a). Although experimental in vitro analyses suggested that only MSH2, MLH1, and PMS1 play a major role in regulating the mutation rate in $C$. neoformans (Boyce et al. 2017), recurrent isolate evolution analysis suggested that MSH5 and RAD5 could also influence isolate evolution in patients (Rhodes et al. 2017a). Given that we did not identify Cn14HC nonsense mutations, we looked at the Cn14HC-specific SNPs responsible for nonconservative mutations in these mismatch repair genes. Interestingly, two of these genes contain mutations specific to Cn14HC: PMS1 (CNAG_07955) encodes a protein with a S483P mutation and RAD5 (CNAG_04733) encodes a protein with a P332S mutation. These SNPs were not present in any of the other 391 isolates considered in this study. In contrast, we did not identify specific SNPs responsible for a change in any of these proteins in Cn216 or Cn225. 
A

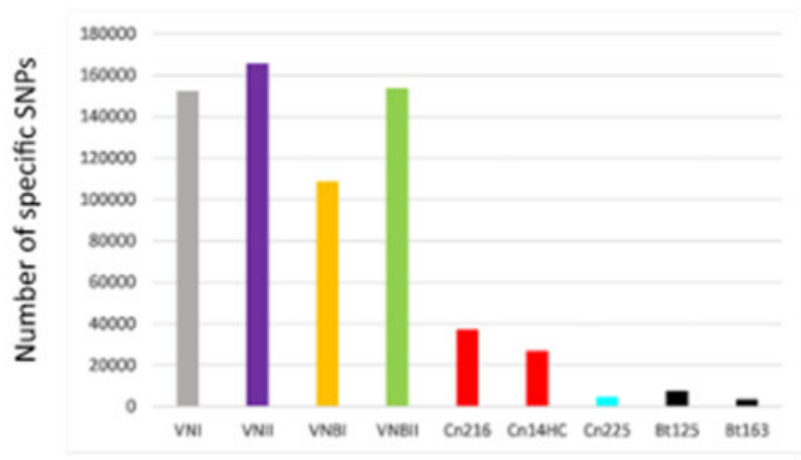

B

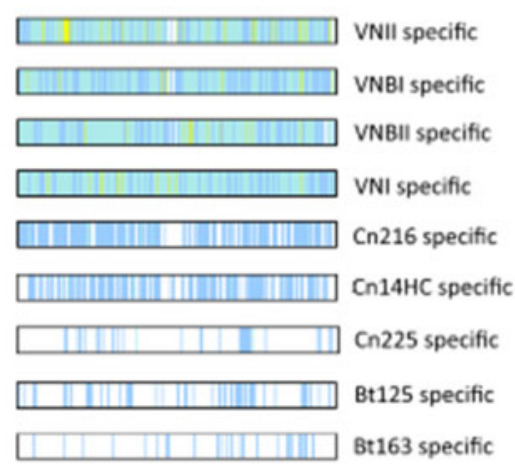

$500 \mathrm{~kb}$ $\begin{array}{llllllll}0 & 5 & 15 & 30 & 50 & 75 & 100 & 150\end{array}$

Figure 4 (A) Number of specific SNPs in each clade and the two Brazilian outlier isolates (Cn216 and Cn14HC). The Brazilian VNBII isolate Cn225 and the hybrid isolates Bt125 and Bt163 (Rhodes et al. 2017b) were used as controls. (B) Density and position of the clade-specific SNPs in the H99 reference genome. The results obtained with chromosome 14 are presented.

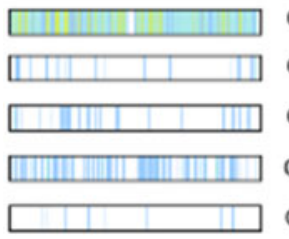

$\operatorname{Cn} 216$

Cn216 ก VNII specific

Cn216 $\cap$ VNBI specific

Cn216 ก VNBII specific

Cn216ก VNI specific
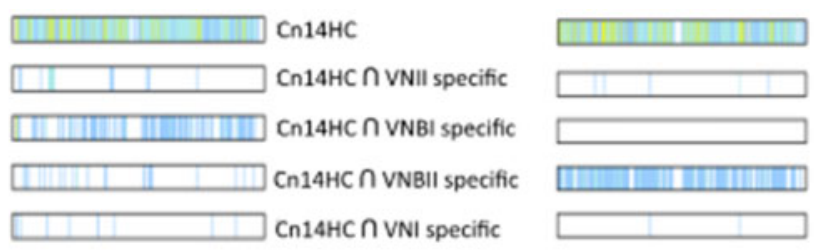

$\operatorname{Cn} 225$

Cn225 ก VNII specific

Cn225 ก VNBI specific

Cn225 ก VNBII specific

Cn225 ก VNI specific

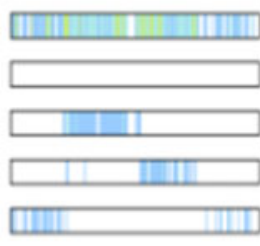

Bt125

Bt125 ก VNII specific

Bt125 ก VNBI specific

Bt125 $\cap$ VNBII specific

Bt125ก VNI specific

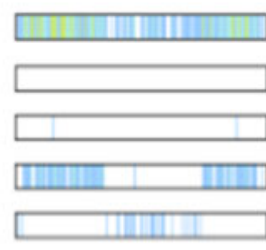

Bt163

Bt163ก VNII specific

Bt163 ก VNBI specific

Bt163ก VNBII specific

Bt163ก VNI specific

$\begin{array}{llllllll}0 & 5 & 15 & 30 & 50 & 75 & 100 & 150\end{array}$

$500 \mathrm{~kb}$

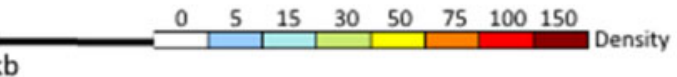

Figure 5 Origin of the SNPs in the Brazilian outlier isolates (Cn216 and Cn14HC). Positions of the SNPs shared between the clade-specific SNPs were identified and plotted on each chromosome. Chromosome 14 is presented as an example. Results obtained with the Brazilian VNBII Cn225 isolate and the African VNB/VNI hybrid isolates Bt125 and Bt163 (Rhodes et al. 2017b) are shown as controls.

Overall, our results suggest that $\mathrm{CnH} 14 \mathrm{C}$ possesses a mutator phenotype associated with specific mutations in two mismatch repair genes. Of course, more experiments are needed to formally establish a causal relationship between this genotype and this phenotype. Nevertheless, the mutator phenotype of $\mathrm{CnH} 14 \mathrm{C}$ has resulted in its rapid evolution, and could explain its position in the phylogenic tree. In contrast, Cn216 might be the result of a long evolution independent of the African VNBII isolate due to an ancient geographical separation.

\section{VNI isolates}

As stated above, nearly all C. neoformans isolates in this collection belong to the VNI clade, mostly within the VNIa subclade. Only two isolates were identified as VNIb and none were identified as VNIc. Interestingly, the second major lineage within the VNIa Brazilian isolates was VNIa_Y, encompassing $27.5 \%(n=10)$ of isolates. All of these strains were isolated from pigeon droppings in Northern Brazil. These are the first VNIa_Y strains isolated outside of Africa; previous strains, all of which originated in the environment, were isolated in Botswana, Malawi, and Uganda (Ashton et al. 2019). The population structure of the Brazilian VNIa isolates resembles that of the African isolates. Approximately $45 \%$ of the VNIa isolates are of the VNIa_93 lineage, similar to previous observations in Ugandan isolates (Ashton et al. 2019). Similar to previous reports for African countries, only 2 of the Brazilian isolates were of the VNIa_5 subclass and no VNIa_4 isolates were identified, confirming that these two subclasses are specifically enriched within Southeast Asia isolates (Ashton et al. 2019).

Phylogenetic trees constructed using only the VNIa subclades revealed a clear geographical clustering of the isolates (Figure 7), suggesting that the current subclade organization of C. neoformans isolates predates the dissemination into South America. However, the existence of unclustered VNI-93 Brazilian isolates suggests multiple intercontinental isolate transfers. 
A

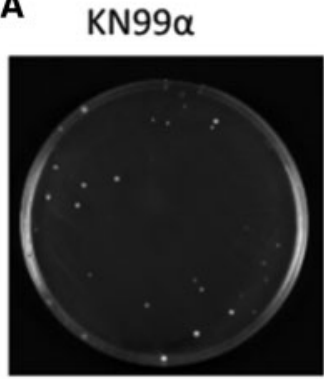

Cn216

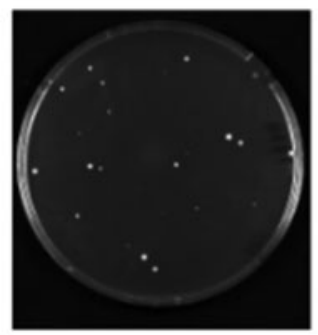

$\mathrm{Cn} 225$

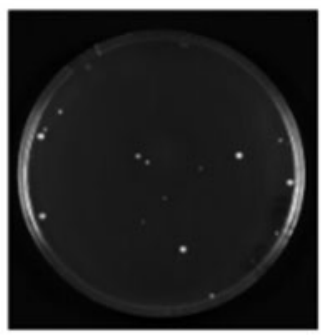

\section{$\mathrm{CnH} 14 \mathrm{C}$}

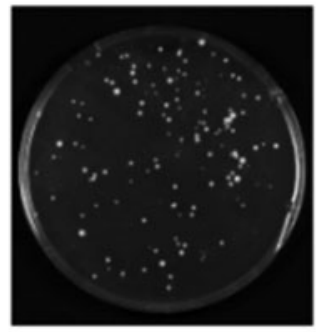

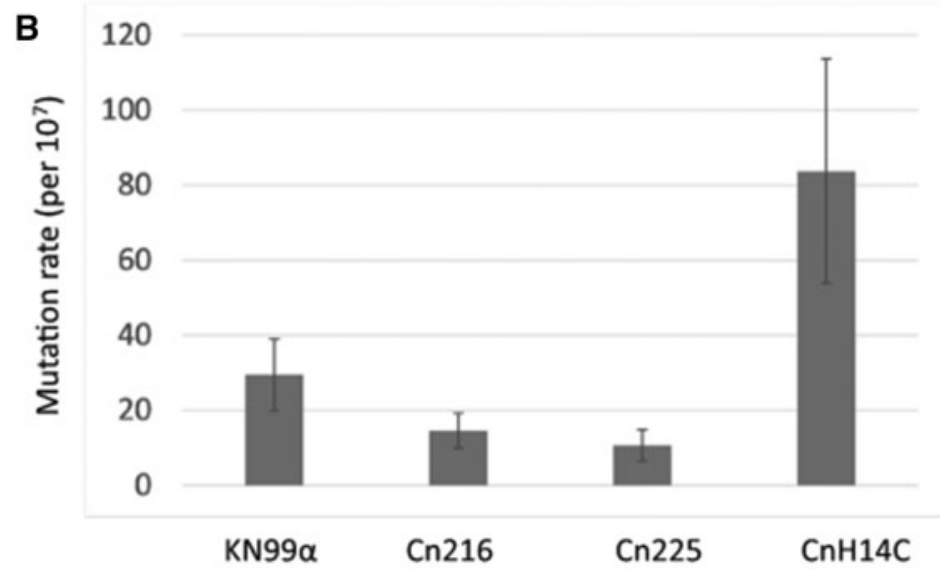

Figure 6 CnH14C displays an elevated mutation rate. (A) Spontaneous 5-FU-resistant colonies in a standard wild type (KN99 $\alpha$ ) isolate and three Brazilian isolates (Cn216, Cn225, and CnH14C). (B) Quantitative assessment of mutation rates in the four strains using fluctuation analysis of the spontaneous resistance to 5-FU. The Lea-Coulson method of the median was used to estimate the number of mutations from the observed values of mutants from 10 independent, parallel cultures (Foster 2006; Gillet-Markowska et al. 2015). Error bars indicate 95\% confidence intervals.
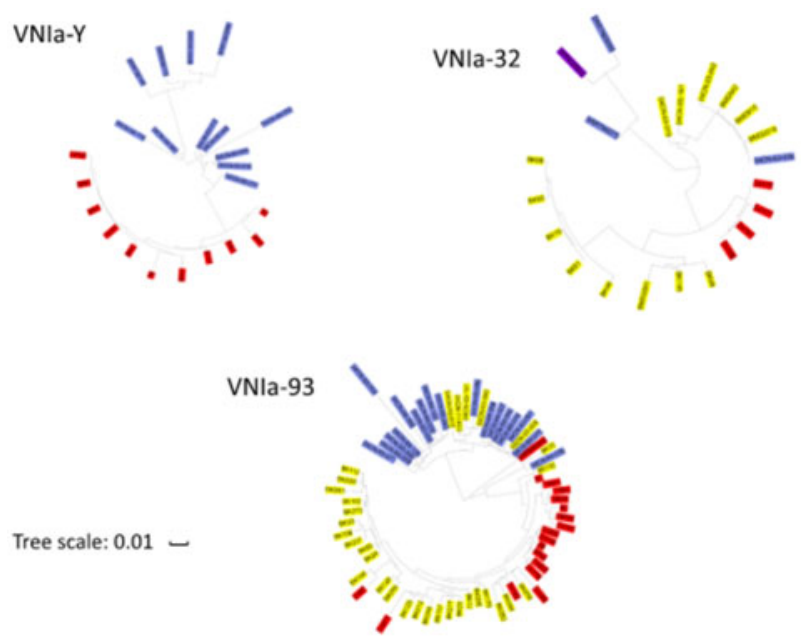

Figure 7 Phylogenetic trees of the VNIa subclades as visualized by ITOL revealed geographic clustering. The Brazilian, African, and South Asian isolates are labeled in red, blue, and yellow, respectively. The Indian isolates are labeled in purple.

\section{Phenotypic analyses}

We next characterized the Brazilian isolates at a phenotypic level. We focused our analysis on virulence-associated phenotypes and drug resistance. All isolates grew well at $30^{\circ} \mathrm{C}$, and only C. gattii isolate Cg187 and C. neoformans VNIa_Y isolate 3Pb3 exhibited a growth defect at $37^{\circ} \mathrm{C}$ (Supplementary Figure S3). Similarly, most of these Cryptococcus isolates demonstrated a low MIC for fluconazole (Supplementary Table S3), with a $\mathrm{MIC}_{50}$ of $2 \mu \mathrm{g} / \mathrm{ml}$ and $\mathrm{MIC}_{90}$ of
$8 \mu \mathrm{g} / \mathrm{ml}$. The clinical isolate Cn191 (VNIa_93) and the environmental isolate 151A1 (VNIa_Y) showed the highest MIC values $(8.0 \mu \mathrm{g} / \mathrm{ml})$.

Capsule formation and exportation of GXM, a major capsular antigen, are essential for Cryptococcus virulence. We analyzed multiple aspects of capsule formation in Brazilian isolates, including capsular morphology, serological reactivity, and extracellular concentration of GXM. Capsule morphology was examined by fluorescence microscopy after incubation of the cells with an antibody to GXM for capsule visualization and calcofluor white for cell wall staining. Alternatively, the capsule was counterstained with India ink for visualization of the capsule by light microscopy. This analysis revealed formidable heterogeneity at morphological and serological levels (Figure 8).

On the basis of the visual perception that antibody recognition of the capsule was variable, we measured the indices of fluorescence intensity after incubation of each isolate with the GXM-binding antibody by flow cytometry (Figure 8B). Using the standard isolates H99 and R265 as reference strains, this analysis confirmed both highly reactive isolates and poorly reactive isolates, supporting the notion that surface GXM is highly diverse in Cryptococcus. Although C. gattii and $C$. deuterogattii isolates were predominantly less capsulated than C. neoformans (student t-test $P$-value $<0.05$ ), we did not observe any statistical difference between the $C$. neoformans subclades.

We also analyzed the concentration of polysaccharide in culture supernatants of each isolate (Supplementary Figure S4). Detection of extracellular and surface-associated GXM showed an apparent correlation, as suggested by the statistical analysis (Supplementary Figure S4), supporting the notion that cryptococcal isolates are highly variable in their ability to form GXM-containing capsules and to export polysaccharide. 
A
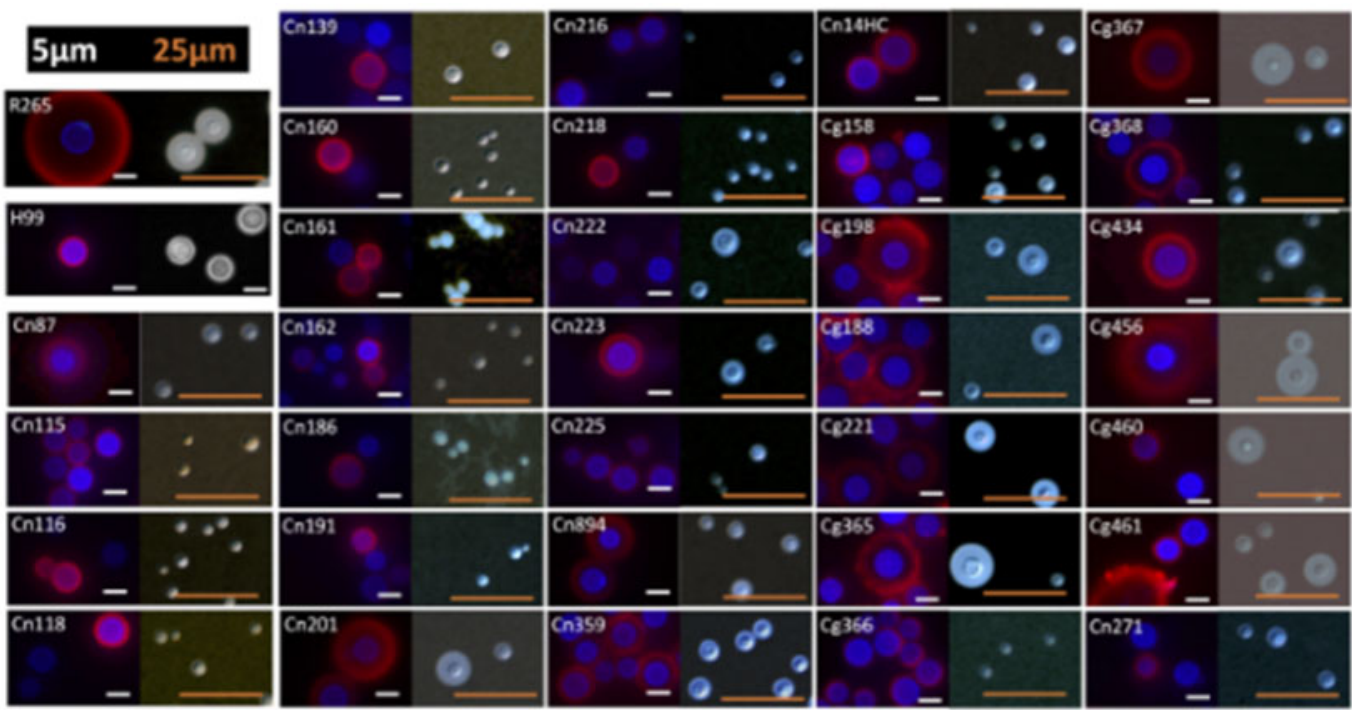

B

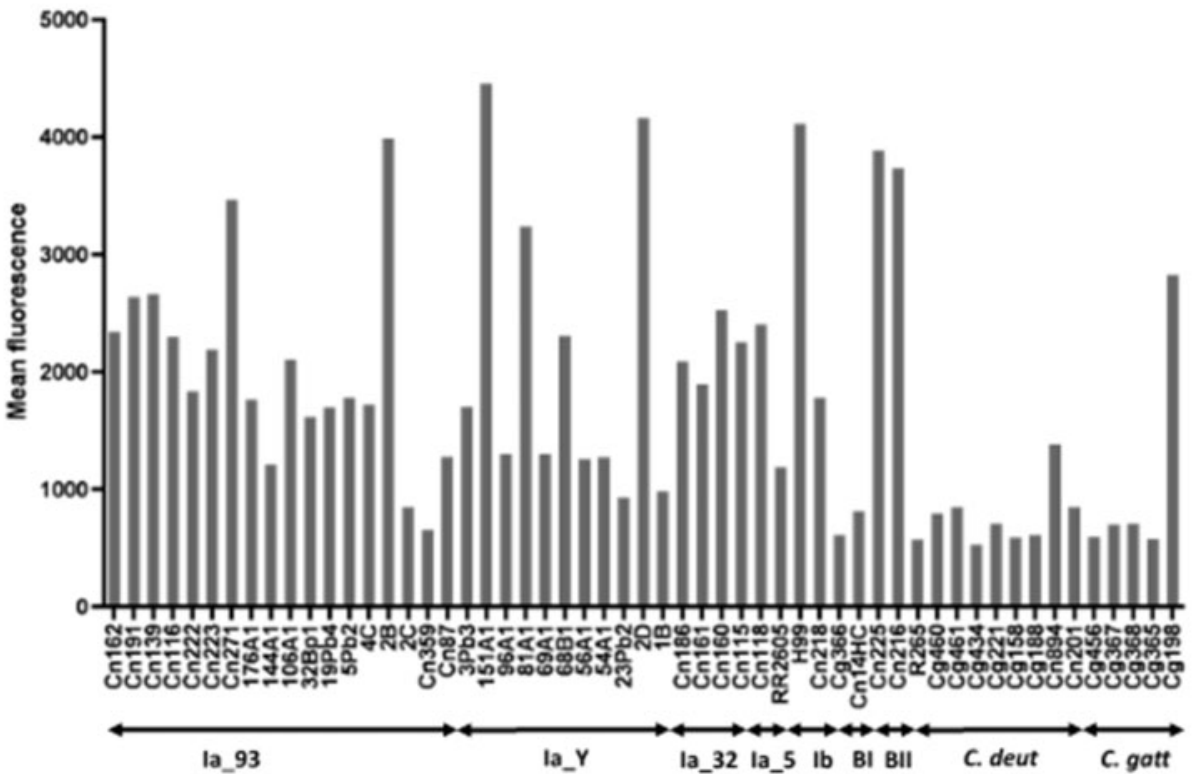

Figure 8 (A) Microscopic analysis of the cell surface of the Brazilian isolates. R265 and H99 are standard cryptococcal isolates that were used as a reference in this assay. For each isolate, fluorescence panels are shown on the left and India ink counterstaining is shown on the right. Red fluorescence indicates capsule staining with the mAb $18 \mathrm{~B} 7$ antibody and blue fluorescence represents cell wall staining. Images are illustrative of two independent analyses that produced similar results. Corresponding data for the Northern Brazil isolates have been previously published (Dal Pupo et al. 2019). (B) Flow cytometry analysis of the cryptococcal isolates after capsule staining with mAb 18B7. At least 5000 cells were analyzed for each isolate. Results are presented as average values of fluorescence intensity and are illustrative of two independent analyses producing similar results. R265 and H99 are standard cryptococcal isolates that were used as a reference in this assay. Data for the Northern Brazil isolates were obtained from Dal Pupo et al. (2019).

We also examined melanin production on L-Dopa medium. We observed a variable phenotype among the Brazilian isolates, with C. gattii and C. deuterogattii isolates displaying lower melanin production (Figure 9). There was no obvious correlation between melanin production among subclades, although strains isolated from bird droppings appear to produce more melanin than other environmental or clinical isolates.

Lastly, we examined the production of TCs. Cryptococcus neoformans and C. gattii cells can undergo an unusual transition in the host lung from the typical size of 5 to $7 \mu \mathrm{m}$ to between $10 \mu \mathrm{m}$ and $100 \mu \mathrm{m}$, a phenomenon known as titanization. TCs are known to be highly polyploid (Zaragoza and Nielsen 2013). TCs can also be induced in vitro using three different approaches (Dambuza et al. 2018; Hommel et al. 2018; Trevijano-Contador et al. 2018). Here, we followed the protocol described by Dambuza et al. (2018) to study whether Brazilian Cryptococcus isolates produce TCs. In our study, C. neoformans $\mathrm{H} 99$ formed nearly 17\% TCs, aligning with previously published findings (Dambuza et al. 2018). Approximately 49\% of Brazilian Cryptococcus isolates produced TCs (defined here as cells with a size $>10 \mu \mathrm{m}$ ) (Figure 10, Supplementary Table S4). Around $24 \%$ of Brazilian Cryptococcus isolates generated more TCs than C. neoformans H99 (Figure 10, Supplementary Table S4). Interestingly, and in contrast to a previous study using the same protocol, all Brazilian C. gattii and C. deuterogattii isolates tested formed TCs (Figure 10, Supplementary Table S4). The highest percentage of TCs (97.33\%) was produced by C. deuterogattii isolate Cn894 (Figure 10, Supplementary Table S4).

In order to address whether cells that formed more TCs than H99 are naturally larger, we compared the size of these cells before and after the titanization induction. The mean size of the 


\section{C. neoformans}

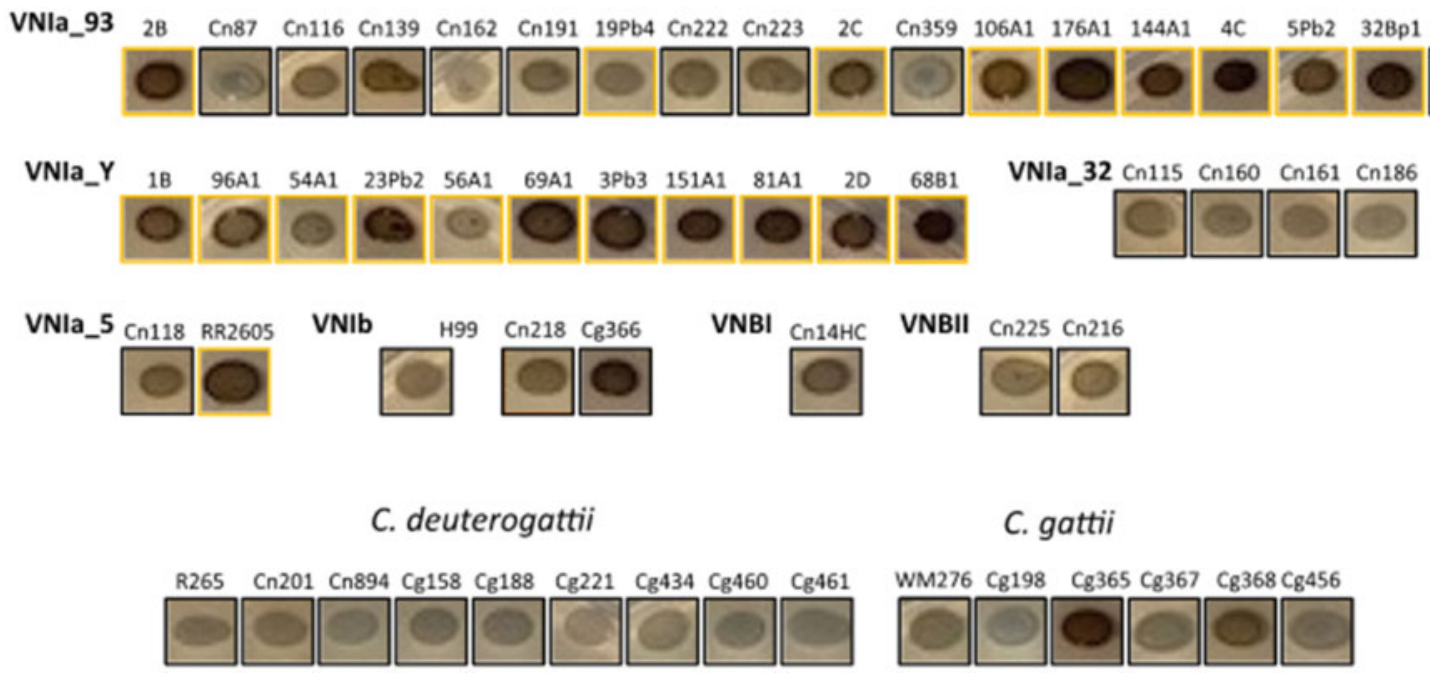

Figure 9 Melanin production of Brazilian isolates as estimated on L-Dopa medium after 72 hours of incubation. Strains isolated from bird droppings are indicated by a yellow box.

A

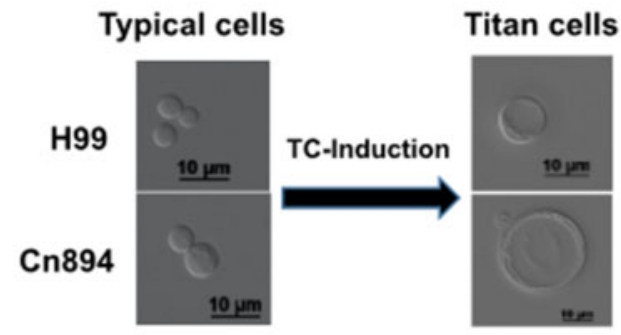

B

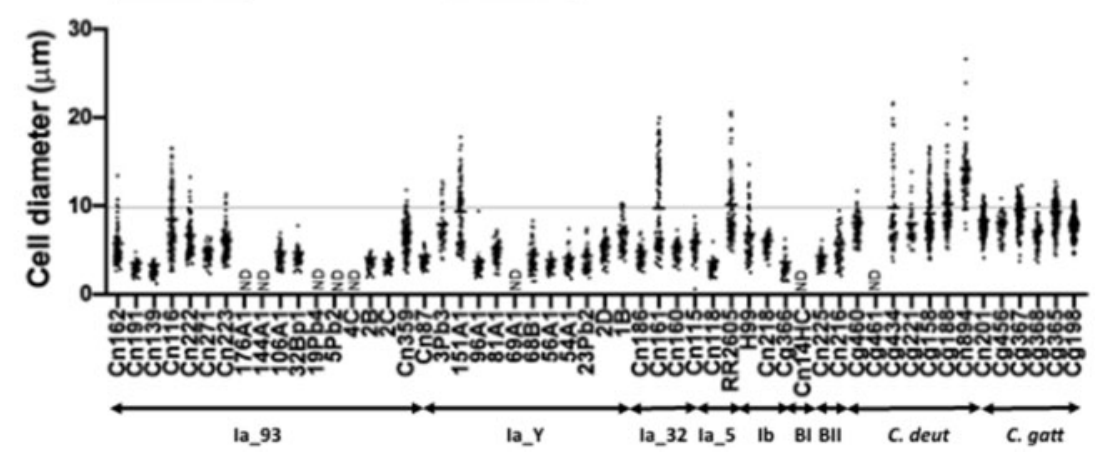

Figure 10 Titanization of Cryptococcus Brazilian isolates (A) Typical examples of the results obtained after TC-induction. (B) Cell diameter after titanization induction in Brazilian isolates. Cryptococcus isolates were subjected to TC induction in PBS-HI-FCS. Cells with a diameter $>10 \mu \mathrm{m}$ were considered to be TCs. ND, not determined.

cells of Brazilian Cryptococcus isolates varied between 4.18 and $5.70 \mu \mathrm{m}$ before and 6.85 to $14.19 \mu \mathrm{m}$ after cultivation in TC-inducing conditions, proving that the titanization induction was responsible for the increase in cell size (Supplementary Table S5). We did not observe any link between subclade and the ability of the corresponding isolate to undergo this phenotypic change.

Overall, this analysis revealed spectacular phenotypic variability within the Brazilian isolates, although no association was detected between one clade and any of these phenotypic traits.

\section{Discussion}

Population genomics studies have resulted in a model in which clonal reproduction has shaped the global population structure of C. neoformans isolates, which are mostly of the VNI clade and the MAT $\alpha$ sexual type (Cuomo et al. 2018). These genome sequence analyses have also identified several VNI subclades with specific geographical distribution (Ashton et al. 2019). Currently, little is known about Cryptococcus species diversity in South America because only a few isolates have been sequenced (Rhodes et al. 2017b). In this study, we analyzed the largest South American genome collection studied to date. As expected, most of the Brazilian isolates belonged to the VNI clade. Our analysis revealed that the VNI Brazilian C. neoformans population shares many features with the African population. For instance, VNI_Y isolates that were previously reported only in Africa were also prominent within this set of South American isolates. As in Africa, Brazilian VNIa_5 isolates were rare; in contrast, VNIa_5 
isolates represent one of the largest subclades within Asian isolates (Ashton et al. 2019). Actually, in most regards, the C. neoformans Brazilian population structure is very different from the Asian one described by Ashton et al. (2019). A detailed analysis of the different VNIa subclade population structures further supported the hypothesis of repeated and privileged exchanges from African to South American isolates. Thus, South American isolates do not form an independent clade or subclade but rather are scattered within nearly all global subclades, indicating repeated transport. Although we observed a perfect clustering of Brazilian isolates within the VNIa_Y and VNIa_32 subcladespossibly from an ancient importation followed by independent evolution of the ancestral isolate-some VNIa-93 isolates clustered with the other global isolates, suggesting independent and possibly more recent importations. Of course, because, all these unclustered VNI-93 Brazilian strains are clinical isolates, we cannot completely exclude the hypothesis that these patients had traveled to Africa, and had been infected there, before developing a cryptococcosis in Brazil as previously described in some rare cases (Garcia-Hermoso et al. 1999).

A similar pattern was observed with the VNB isolates. Two of the Brazilian isolates were positioned close to the roots of the VNB branches, as previously observed for two sequenced Brazilian isolates (Rhodes et al. 2017b). However, the third isolate clustered within the other VNB African isolates, suggesting a more recent intercontinental exchange. In that case, it is important to note that this last strain has been isolated in the environment.

In 2017, Casadevall and colleagues suggested that the separation of C. neoformans complex species and C. gattii complex species occurred in the middle of the Cretaceous period (Casadevall et al. 2017). This geological period was marked by the breakup of the supercontinent west Gondawa which encompassed Africa and South America. This modern continent formation ended much before the global dispersion of major Cryptococcus spreader birds (Prum et al. 2015) which occurred $40 \times 10^{6}$ years after the end of the Cretaceous period. Although our data cannot reliably date the separation between the C. neoformans subclades, the similar structure of the African and South American population suggests an African origin for every C. neoformans isolates. In this model, the transport of C. neoformans from Africa to South America started only with birds migrations $25 \times 10^{6}$ years ago and continued until today.

This study also exemplified the impact of rapidly evolving isolates on the C. neoformans population structure. Mutator phenotypes have already been observed in natural C. neoformans and C. gattii isolates and have been shown to impact the dynamics of isolate evolution, phenotypic diversity, and drug resistance (Billmyre et al. 2017; 2020). A msh2-associated mutator phenotype was also observed in a subset of $\mathrm{C}$. deuterogattii Pacific Northwest outbreak isolates, named VGIIa-like (Kidd et al. 2004; Billmyre et al. 2014, 2017). Here, the VNBI isolate CnH14C displayed a mutator phenotype, albeit less pronounced than the previously described C. neoformans $m s h 2 \Delta$, $m \ln 1 \Delta$, and pms $1 \Delta$ mutant strains (Boyce et al. 2017). Accordingly, we did not identify nonsense mutations in any of these genes in the $\mathrm{CnH} 14 \mathrm{C}$ isolate. However, PMS1- and RAD5-specific alleles in CnH14C might suggest a causal relationship. In fact, RAD5 has been recently identified as regulating mutation rates in a quantitative trait locus analysis in S. cerevisiae (Gou et al. 2019), and a specific RAD5 mutation has also been identified in a rapidly evolved C. neoformans recurrent clinical isolate (Rhodes et al. 2017a).
However, in contrast to what has been observed in some African isolates, we did not identify any hybrid isolates within this collection of Brazilian isolates. As previously reported (Matsumoto et al. 2007; Andrade-Silva et al. 2018), none of these isolates were MATa. Nevertheless, our model of multiple intercontinental transport of African isolates to South America and the proportion of MATa isolate within the VNB isolates in Africa (Litvintseva et al. 2007; 2011; Litvintseva and Mitchell 2012) predict that additional sampling will probably identify such isolates.

MLST and genome sequence analysis identified South America, and in particular Brazil, as the major source of the global genetic diversity of the C. gattii complex, especially within the VGII and VGIII lineages (Hagen et al. 2013; Billmyre et al. 2014; Souto et al. 2016; Barcellos et al. 2018; Vilas-Bôas et al. 2020). This is associated with great phenotypic diversity within the Brazilian isolates (Silva et al. 2012; Barcellos et al. 2018). Similarly, genotypic and phenotypic analyses of Brazilian C. neoformans isolates, including the analysis in this study, have revealed great diversity within South American isolates (Ferreira-Paim et al. 2017; Andrade-Silva et al. 2018; Dal Pupo et al. 2019; De Sousa et al. 2020). Nevertheless, our data suggest that Brazil is probably not an important source of global diversity of C. neoformans isolates. Rather, our findings support the model in which this diversity is mostly imported from Africa through repeated intercontinental transport of C. neoformans isolates to South America.

Our studies continue the investigation of Cryptococcus species global diversity and emphasize the importance of understanding the evolution of fungal pathogens. Further studies with additional isolates from Brazil and other countries in South America will help us to clarify many aspects of the population genetics of this important fungal pathogen.

\section{Acknowledgments}

M.L.R. is currently on leave from the position of associate professor at the Microbiology Institute of the Federal University of Rio de Janeiro, Brazil.

\section{Funding}

M.L.R., G.J., and G.H.G. were supported by a Tripartite USPFiocruz-Pasteur grant. FCGR was financed in part by a scholarship from the Coordenação de Aperfeiçoamento de Pessoal de Nível Superior (CAPES, Brazil, Finance Code 001). This work in the Janbon lab was supported in part by an Infect-ERA grant (project Cryptoview).

Conflicts of interest: None declared.

\section{Literature cited}

Alanio A. 2020. Dormancy in Cryptococcus neoformans: 60 years of accumulating evidence. J Clin Invest. 130:3353-3360.

Aminnejad M, Diaz M, Arabatzis M, Castaneda E, Lazera M, et al. 2012. Identification of novel hybrids between Cryptococcus neoformans var. grubii VNI and Cryptococcus gattii VGII. Mycopathologia. 173:337-346.

Andrade-Silva LE, Ferreira-Paim K, Ferreira TB, Vilas-Boas A, Mora DJ, et al. 2018. Genotypic analysis of clinical and environmental Cryptococcus neoformans isolates from Brazil reveals the presence of VNB isolates and a correlation with biological factors. PLoS One. 13:e0193237. 
Ashton PM, Thanh LT, Trieu PH, Van Anh D, Trinh NM, et al. 2019. Three phylogenetic groups have driven the recent population expansion of Cryptococcus neoformans. Nat Commun. 10:2035.

Barcellos VA, Martins LMS, Fontes ACL, Reuwsaat JCV, Squizani ED, et al. 2018. Genotypic and phenotypic diversity of Cryptococcus gattii VGII Clinical isolates and its impact on virulence. Front Microbiol. 9:132-132.

Basenko EY, Pulman JA, Shanmugasundram A, Harb OS, Crouch K, et al. 2018. FungiDB: an integrated bioinformatic resource for fungi and oomycetes. J Fungi (Basel). 4:39.

Billmyre RB, Applen Clancey S, Li LX, Doering TL, Heitman J. 2020. 5 -fluorocytosine resistance is associated with hypermutation and alterations in capsule biosynthesis in Cryptococcus. Nat Commun. 11:127. [10.1038/s41467-019-13890-z]

Billmyre RB, Clancey SA, and, Heitman J. 2017. Natural mismatch repair mutations mediate phenotypic diversity and drug resistance in Cryptococcus deuterogattii. eLife. 6:e28802.

Billmyre RB, Croll D, Li W, Mieczkowski P, Carter DA, et al. 2014. Highly recombinant VGII Cryptococcus gattii population develops clonal outbreak clusters through both sexual macroevolution and asexual microevolution. mBio. 5:e01494-14.

Boekhout T, Theelen B, Diaz M, Fell JW, Hop WCJ, et al. 2001. Hybrid genotypes in the pathogenic yeast Cryptococcus neoformans. Microbiology (Reading). 147:891-907.

Bovers M, Hagen F, Kuramae EE, Hoogveld HL, Dromer F, et al. 2008. AIDS patient death caused by novel Cryptococcus neoformans $\mathrm{x}$ C. gattii hybrid. Emerg Infect Dis. 14:1105-1108.

Boyce KJ, Wang Y, Verma S, Shakya VPS, Xue C, et al. 2017. Mismatch repair of DNA replication errors contributes to microevolution in the pathogenic fungus Cryptococcus neoformans. mBio. 8: e00595-17.

Bui T, Lin X, Malik R, Heitman J, Carter D. 2008. Isolates from Cryptococcus neoformans from infected animals reveal genetics exchange in unisexual, a mating type population. Eukaryot Cell. 7: $1771-1780$

Casadevall A, Freij JB, Hann-Soden C, and, Taylor J. 2017. Continental drift and speciation of the Cryptococcus neoformans and Cryptococcus gattii species complexes. mSphere. 2:e00103-17.

Casadevall A, Mukherjee J, Devi SJN, Schneerson R, Robbins JB, et al. 1992. Antibodies elicited by a Cryptococcus neoformans-tetanus toxoid conjugate vaccine have the same specificity as those elicited in infection. J Infect Dis. 165:1086-1093.

Clinical Laboratory Standards Institute. 2017. Reference method for broth dilution antifungal susceptibility testing of yeasts; approved standard, document. Document M27-A3 in National Committee for Clinical Laboratory Standards.

Cuomo CA, Rhodes J, Desjardins CA. 2018. Advances in Cryptococcus genomics: insights into the evolution of pathogenesis. Mem Inst Oswaldo Cruz. 113:e170473

Dal Pupo HD, Sena BAG, Reis FCG, Machado L, Fortes ST, et al. 2019. Polysaccharide diversity in VNI isolates of Cryptococcus neoformans from Roraima, Northern Brazil. Fungal Biol. 123:699-708.

Dambuza IM, Drake T, Chapuis A, Zhou X, Correia J, et al. 2018. The Cryptococcus neoformans Titan cell is an inducible and regulated morphotype underlying pathogenesis. PLoS Pathog. 14:e1006978.

Desjardins CA, Giamberardino C, Sykes SM, Yu C-H, Tenor JL, et al. 2017. Population genomics and the evolution of virulence in the fungal pathogen Cryptococcus neoformans. Genome Res. 27: 1207-1219.

de Sousa HR, de Oliveira GP, de Oliveira Frazão S, de Melo Gorgonha KC, Rosa CP, et al. 2020. Faster Cryptococcus melanization increases virulence in experimental and human cryptococcosis. bioRxiv. 2020.2007.2029.222794
Engelthaler DM, Hicks ND, Gillece JD, Roe CC, Schupp JM, et al. 2014. Cryptococcus gattii in North American Pacific Northwest: whole-population genome analysis provides insights into species evolution and dispersal. mBio. 5:e01464-14.

Ergin C,, Şengül M, Aksoy L, Döğen A, Sun S, et al. 2019. Cryptococcus neoformans recovered from olive trees (Olea europaea) in Turkey reveal allopatry with African and South American lineages. Front Cell Infect Microbiol. 9:384

Farrer RA, Chang M, Davis MJ, van Dorp L, Yang D-H, et al. 2019. A new lineage of Cryptococcus gattii (VGV) discovered in the central Zambezian Miombo Woodlands. mBio. 10:e02306-19.

Ferreira-Paim K, Andrade-Silva L, Fonseca FM, Ferreira TB, Mora DJ, et al. 2017. MLST-based population genetic analysis in a global context reveals clonality amongst Cryptococcus neoformans var. grubii VNI isolates from HIV patients in Southeastern Brazil. PLoS Negl Trop Dis. 11:e0005223.

Foster PL. 2006. Methods for determining spontaneous mutation rates. Methods Enzymol. 409:195-213.

Garcia-Hermoso D, Janbon G, Dromer F. 1999. Epidemiological evidence for dormant Cryptococcus neoformans infection. J Clin Microbiol. 37:3204-3209.

Gillet-Markowska A, Louvel G, Fischer G. 2015. bz-rates: a web tool to estimate mutation rates from fluctuation analysis. G3 (Bethesda). 5:2323-2327.

Gonzalez-Hilarion S, Paulet D, Lee K-T, Hon C-C, Lechat P, et al. 2016. Intron retention-dependent gene regulation in Cryptococcus neoformans. Sci Rep. 6:32252.[10.1038/srep32252]

Gou L, Bloom JS, Kruglyak L. 2019. The genetic basis of mutation rate variation in yeast. Genetics. 211:731-740.

Gröhs Ferrareze PA, Maufrais C, Silva Araujo Streit R, Priest SJ, Cuomo CA, et al. 2021. Application of an optimized annotation pipeline to the Cryptococcus deuterogattii genome reveals dynamic primary metabolic gene clusters and genomic impact of RNAi loss. G3 (Bethesda). 11:jkaa070.[10.1093/g3journal/jkaa070]

Guindon S, Dufayard J-F, Lefort V, Anisimova M, Hordijk W, et al. 2010. New algorithms and methods to estimate maximum-likelihood phylogenies: assessing the performance of PhyML 3.0. Syst Biol. 59:307-321.

Hagen F, Ceresini PC, Polacheck I, Ma H, Nieuwerburgh F. V, et al. 2013. Ancient dispersal of the human fungal pathogen Cryptococcus gattii from the Amazon rainforest. PLoS One. 8: e71148.

Hagen F, Khayhan K, Theelen B, Kolecka A, Polacheck I, et al. 2015. Recognition of seven species in the Cryptococcus gattii/Cryptococcus neoformans species complex. Fungal Genet Biol. 78:16-48.

Hommel B, Mukaremera L, Cordero RJB, Coelho C, Desjardins CA, et al. 2018. Titan cells formation in Cryptococcus neoformans is finely tuned by environmental conditions and modulated by positive and negative genetic regulators. PLoS Pathog. 14:e1006982.

Janbon G, Ormerod KL, Paulet D, Byrnes EJ, Yadav V, et al. 2014. Analysis of the genome and transcriptome of Cryptococcus neoformans var. grubii reveals complex RNA expression and microevolution leading to virulence attenuation. PLoS Genet. 10:e1004261.

Janbon G, Quintin J, Lanternier F, d'Enfert C. 2019. Studying fungal pathogens of humans and fungal infections: fungal diversity and diversity of approaches. Genes Immun. 20:403-414

Johnston SA, Voelz K, May RC. 2016. Cryptococcus neoformans thermotolerance to avian body temperature is sufficient for extracellular growth but not intracellular survival in macrophages. Sci Rep. 6: 20977. [10.1038/srep20977]

Kidd SE, Hagen F, Tscharke RL, Huynh M, Bartlett KH, et al. 2004. A rare genotype of Cryptococcus gattii caused the cryptococcosis 
outbreak on Vancouver Island (British Columbia, Canada). Proc Natl Acad Sci USA. 101:17258-17263.

Kwon-Chung KJ, Bennett JE. 1978. Distribution of a and a mating types of Cryptococcus neoformans among natural and clinical isolates. Am J Epidemiol. 108:337-340.

Kwon-Chung KJ, Fraser JA, Doering TL, Wang Z, Janbon G, et al. 2014. Cryptococcus neoformans and Cryptococcus gattii, the etiologic agents of cryptococcosis. Cold Spring Harb Perspect Med. 4:a019760.

Li H. 2018. Minimap2: pairwise alignment for nucleotide sequences. Bioinformatics. 34:3094-3100.

Li H, Handsaker B, Wysoker A, Fennell T, Ruan J, et al. 2009. The Sequence Alignment/Map format and SAMtools. Bioinformatics. 25:2078-2079.

Lin X, Heitman J. 2006. The biology of the Cryptococcus neoformans species complex. Annu Rev Microbiol. 60:69-105.

Lin X, Patel S, Litvintseva AP, Floyd A, Mitchell TG, et al. 2009. Diploids in the Cryptococcus neoformans serotype A population homozygous for the alpha mating type originate via unisexual mating. PLoS Pathog. 5:e1000283.

Litvintseva AP, Carbone I, Rossouw J, Thakur R, Govender NP, et al. 2011. Evidence that the human pathogenic fungus Cryptococcus neoformans var. grubii may have evolved in Africa. PLoS One. 6: e19688.

Litvintseva AP, Kestenbaum L, Vilgalys R, Mitchell TG. 2005. Comparative analysis of environmental and clinical populations of Cryptococcus neoformans. J Clin Microbiol. 43:556-564.

Litvintseva AP, Lin X, Templeton I, Heitman J, and, Mitchell TG. 2007. Many globally isolated AD hybrid strains of Cryptococcus neoformans originated in Africa. PLoS Pathog. 3:e114.

Litvintseva AP, Marra RE, Nielsen K, Heitman J, Vilgalys R, et al. 2003. Evidence of sexual recombination among Cryptococcus neoformans serotype A isolates in sub-Saharan Africa. Eukaryot Cell. 2: 1162-1168.

Litvintseva AP, Mitchell TG. 2012. Population genetic analyses reveal the African origin and strain variation of Cryptococcus neoformans var. grubii. PLoS Pathog. 8:e1002495.

Loftus B, Fung E, Roncaglia P, Rowley D, Amedeo P, et al. 2005. The genome and transcriptome of Cryptococcus neoformans, a basidiomycetous fungal pathogen of humans. Science. 307:1321-1324.

Matsumoto MT, Fusco-Almeida AM, Baeza LC, Melhem MDSC, Medes-Giannini MJS. 2007. Genotyping, serotyping and determination of mating-type of Cryptococcus neoformans clinical isolates from São Paulo State, Brazil. Rev Inst Med Trop Sao Paulo. 49: 41-47.

Meyer W, Aanensen DM, Boekhout T, Cogliati M, Diaz MR, et al. 2009. Consensus multi-locus sequence typing scheme for Cryptococcus neoformans and Cryptococcus gattii. Med Mycol. 47:561-570.
Prum RO, Berv JS, Dornburg A, Field DJ, Townsend JP, et al. 2015. A comprehensive phylogeny of birds (Aves) using targeted nextgeneration DNA sequencing. Nature. 526:569-573.

Raj A, Stephens M, Pritchard JK. 2014. fastSTRUCTURE: variational inference of population structure in large SNP data sets. Genetics. 197:573-589.

Rajasingham R, Smith RM, Park BJ, Jarvis JN, Govender NP, et al. 2017. Global burden of disease of HIV-associated cryptococcal meningitis: an updated analysis. Lancet Infect Dis. 17:873-881.

Rhodes J, Beale MA, Vanhove M, Jarvis JN, Kannambath S, et al. 2017a. A population genomics approach to assessing the genetic basis of within-host microevolution underlying recurrent cryptococcal meningitis infection. G3 (Bethesda). 7:1165-1176.

Rhodes J, Desjardins CA, Sykes SM, Beale MA, Vanhove M, et al. 2017b. Tracing genetic exchange and biogeography of Cryptococcus neoformans var. grubii at the global population level. Genetics. 207:203836-203346.

Ruma P, Chen SCA, Sorrel TC, Brownlee AG. 1996. Characterization of Cryptococcus neoformans by random DNA amplification. Lett Appl Microbiol. 23:312-316.

Silva DC, Martins MA, Szeszs MW, Bonfietti LX, Matos D, et al. 2012. Susceptibility to antifungal agents and genotypes of Brazilian clinical and environmental Cryptococcus gattii strains. Diagn Microbiol Infect Dis. 72:332-339.

Souto ACP, Bonfietti LX, Ferreira-Paim K, Trilles L, Martins M, et al. 2016. Population genetic analysis reveals a high genetic diversity in the Brazilian Cryptococcus gattii VGII population and shifts the global origin from the amazon rainforest to the semi-arid desert in the Northeast of Brazil. PLoS Negl Trop Dis. 10:e0004885.

Trevijano-Contador N, de Oliveira HC, García-Rodas R, Rossi SA, Llorente I, et al. 2018. Cryptococcus neoformans can form titan-like cells in vitro in response to multiple signals. PLoS Pathog. 14: e1007007.

Vanhove M, Beale MA, Rhodes J, Chanda D, Lakhi S, et al. 2017. Genomic epidemiology of Cryptococcus yeasts identifies adaptation to environmental niches underpinning infection across an African HIV/AIDS cohort. Mol Ecol. 26:1991-2005.

Vilas-Bôas AM, Andrade-Silva LE, Ferreira-Paim K, Mora DJ, Ferreira TB, et al. 2020. High genetic variability of clinical and environmental Cryptococcus gattii isolates from Brazil. Med Mycol. 58: 1126-1137.

Yadav V, Sun S, Billmyre RB, Thimmappa BC, Shea T, et al. 2018. RNAi is a critical determinant of centromere evolution in closely related fungi. Proc Natl Acad Sci USA. 115:3108-3113.

Zaragoza O, Nielsen K. 2013. Titan cells in Cryptococcus neoformans: cells with a giant impact. Curr Opin Microbiol. 16:409-413.

Communicating editor: A. Rokas 